Supplement of Hydrol. Earth Syst. Sci., 24, 945-966, 2020

https://doi.org/10.5194/hess-24-945-2020-supplement

(c) Author(s) 2020. This work is distributed under

the Creative Commons Attribution 4.0 License.

(c) (1)

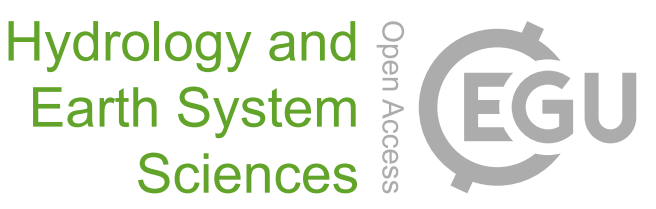

Supplement of

\title{
Temperature controls production but hydrology regulates export of dissolved organic carbon at the catchment scale
}

\section{Hang Wen et al.}

Correspondence to: Li Li (lili@engr.psu.edu)

The copyright of individual parts of the supplement might differ from the CC BY 4.0 License. 
S1. Estimation of groundwater flow $\boldsymbol{Q}_{G}$. Based on estimation in Li et al. (2017), groundwater estimates were refined first by calculating average groundwater fluxes in wet and dry periods using the conductivity mass balance hydrograph separation (Lim et al., 2005) via the online Web-based Hydrograph Analysis Tool

35 (WHAT) (https:// engineerg.purdue.edu/ what). The groundwater influx was further refined by capturing the peaks of stream DOC concentration, especially under low discharge periods.

40

45

50

55

60

65

70

75 


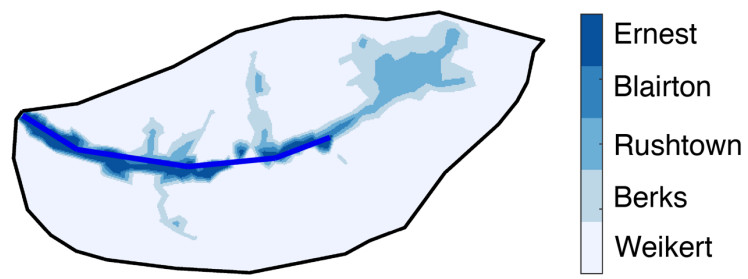

Figure S1. Spatial distribution of soil series at Shale Hills.

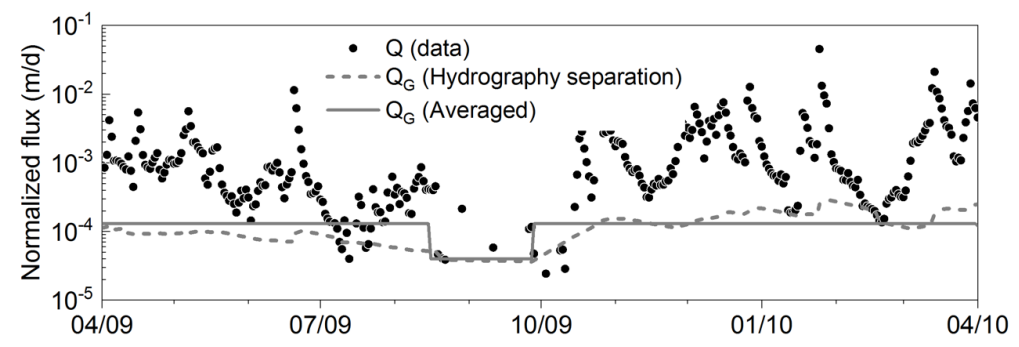

Figure S2. Temporal dynamics of field discharge (dots), groundwater flow $Q_{G}$ estimated from WHAT (dash line), and corresponding averaged $Q_{G}$ in the wet and dry periods (solid line).

95 


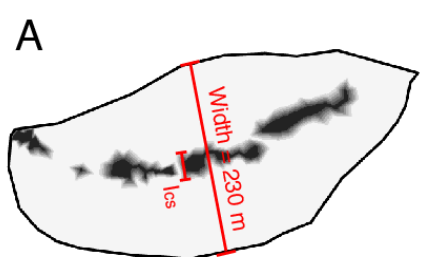

Ics $/$ Width $=0.10$

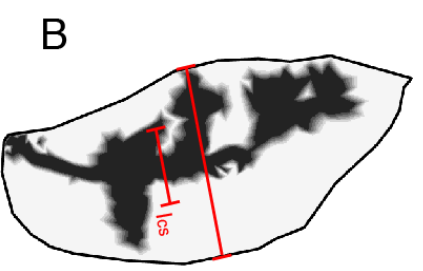

Ics $/$ Width $=0.35$

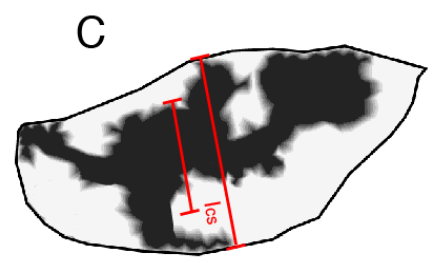

Ics $/$ Width $=0.70$

Figure. S3. Maps showing conditions with different hydrological connectivity $I_{\text {cs }} /$ Width, including (A) 0.10 , (B) 0.35 , and (C) 0.70 .
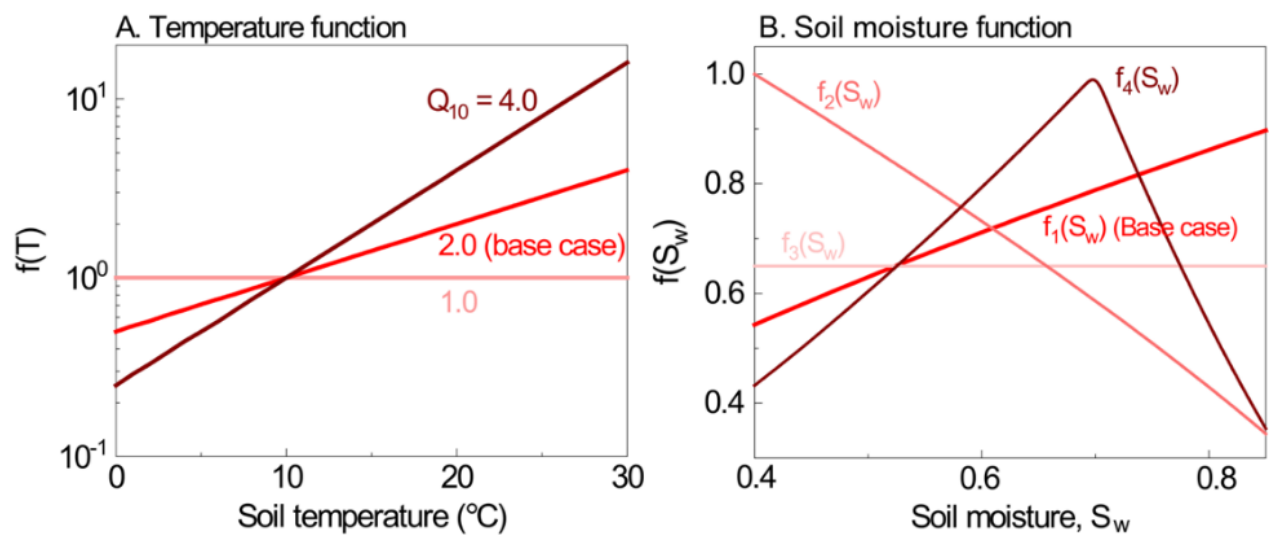

115 Figure. S4. Sensitivity analysis for $(A)$ the temperature function and $(B)$ the four soil moisture functions used to calculate local DOC production rate (Eq. (2)-(5)). The four soil moisture functions represent increase, decrease, constant, and threshold behavior, respectively. 
Table S1. Soil parameters. Listed values are the a priori (uncalibrated) parameter values. All parameters in this table are calibrated using an optimization algorithm.

\begin{tabular}{|c|c|c|c|c|c|c|c|}
\hline \multirow{2}{*}{ Parameter } & \multirow{2}{*}{ Description } & \multicolumn{5}{|c|}{ Soil type } & \multirow{2}{*}{ Source } \\
\hline & & Weikert & Berks & Rushtown & Blairton & Ernest & \\
\hline$K_{\text {infV }}$ & $\begin{array}{l}\text { Vertical saturated hydraulic } \\
\text { conductivity of infiltration layer } \\
(\mathrm{m} / \mathrm{s})\end{array}$ & 9.1 & 15.2 & 9.8 & 1.5 & 8.3 & (Lin, 2006) \\
\hline$K_{V}$ & $\begin{array}{l}\text { Vertical saturated hydraulic } \\
\text { conductivity }(\mathrm{m} / \mathrm{s})\end{array}$ & 1.6 & 1.9 & 1.1 & 0.7 & 3.7 & (Lin, 2006) \\
\hline$K_{H}$ & $\begin{array}{l}\text { Horizontal saturated hydraulic } \\
\text { conductivity }(\mathrm{m} / \mathrm{s})\end{array}$ & 1.2 & 1.0 & 2.3 & 3.0 & 7.0 & (Lin, 2006) \\
\hline$\phi$ & Porosity $\left(\mathrm{m}^{3} / \mathrm{m}^{3}\right)$ & 0.37 & 0.40 & 0.42 & 0.41 & 0.49 & (Lin, 2006) \\
\hline$\dot{\phi}_{r}$ & Residual porosity $\left(\mathrm{m}^{3} / \mathrm{m}^{3}\right)$ & 0.05 & 0.05 & 0.05 & 0.05 & 0.05 & (Lin, 2006) \\
\hline$\alpha$ & Van Genuchten soil parameter $\left(\mathrm{m}^{-1}\right)$ & 8.80 & 6.45 & 6.50 & 5.34 & 5.82 & (Lin, 2006) \\
\hline $\boldsymbol{\beta}$ & Van Genuchten soil parameter (-) & 1.24 & 1.21 & 1.26 & 1.26 & 1.22 & (Lin, 2006) \\
\hline $\begin{array}{l}f_{\text {mac }, V} \text { and } \\
f_{\text {mac }, H}\end{array}$ & $\begin{array}{l}\text { Vertical and horizontal area } \\
\text { fraction of macropores }\left(\mathrm{m}^{2} / \mathrm{m}^{2}\right)\end{array}$ & 0.01 & & & & & $\begin{array}{l}\text { Empirical (Shi } \\
\text { et al., 2013) }\end{array}$ \\
\hline$D_{m a c}$ & Macropore depth (m) & 1.0 & & & & & $\begin{array}{l}\text { Empirical (Shi } \\
\text { et al., 2013) }\end{array}$ \\
\hline$K_{m a c, V}$ & $\begin{array}{l}\text { Vertical macropore hydraulic } \\
\text { conductivity }(\mathrm{m} / \mathrm{s})^{\mathrm{a}}\end{array}$ & $100 K_{\text {infV }}$ & & & & & $\begin{array}{l}\text { Empirical (Shi } \\
\text { et al., 2013) }\end{array}$ \\
\hline$K_{m a c, H}$ & $\begin{array}{l}\text { Horizontal macropore hydraulic } \\
\text { conductivity }(\mathrm{m} / \mathrm{s})^{\mathrm{a}}\end{array}$ & $1000 K_{H}$ & & & & & $\begin{array}{l}\text { Empirical (Shi } \\
\text { et al., 2013) }\end{array}$ \\
\hline
\end{tabular}

a. Soil horizontal macropore hydraulic conductivity and soil vertical macropore hydraulic conductivity are assumed to be 1000 and 100 times their corresponding soil matrix conductivities, respectively.

\section{Reference}

Li, L., Bao, C., Sullivan, P. L., Brantley, S., Shi, Y., and Duffy, C.: Understanding watershed hydrogeochemistry: 2. Synchronized hydrological and geochemical processes drive stream chemostatic behavior, Water Resour Res, 2017.

140 Lim, K. J., Engel, B. A., Tang, Z. X., Choi, J., Kim, K. S., Muthukrishnan, S., and Tripathy, D.: Automated Web Gis based hydrograph analysis tool, what, Journal of the American Water Resources Association, 41, 1407-1416, 10.1111/j.1752-1688.2005.tb03808.x, 2005. Lin, H.: Temporal stability of soil moisture spatial pattern and subsurface preferential flow pathways in the shale hills catchment, Vadose Zone J, 5, 317-340, 10.2136/vzj2005.0058, 2006.

145 Shi, Y. N., Davis, K. J., Duffy, C. J., and Yu, X.: Development of a Coupled Land Surface Hydrologic Model and Evaluation at a Critical Zone Observatory, Journal of Hydrometeorology, 14, 1401-1420, 10.1175/jhm-d-12-0145.1, 2013. 